\title{
Implantação da Comissão da Qualidade e Segurança do Paciente em Instituição Hospitalar: relato de experiência
}

\author{
Implantation of the Commission on Quality and Patient Safety in a Hospital Institution: \\ report of experience \\ Implantación de la Comisión de Calidad y Seguridad del Paciente en una Institución \\ Hospitalaria: relato de experiencia
}

Thaisa Cristina Afonso ${ }^{1 *}$, Ana Lúcia Queiroz Bezerra ${ }^{1}$, Leyla Gabriela Verner Amaral Brandão, Robson Tostes Amaral $^{1}$, Cristiane Chagas Teixeira ${ }^{1}$, Adrielle Cristina Silva Souza ${ }^{1}$, Nívea Rodrigues Borges ${ }^{2}$.

\section{RESUMO}

Objetivo: Relatar a experiência da implantação da Comissão da Qualidade e Segurança do Paciente como estratégia para promover a qualidade assistencial. Métodos: Trata-se de um relato de experiência, de abordagem crítico-reflexiva, acerca da implantação da Comissão da Qualidade e Segurança do Paciente em um hospital na região sudoeste de Goiás, no período de 2012 a 2017. Relato de experiência: O estudo demonstra a viabilidade de se adotar a Comissão quanto estratégia às práticas de segurança, principalmente no que tange a melhor interação entre as equipes, gestão de indicadores, e ainda, ao despertar do comprometimento dos profissionais com a execução do serviço no critério qualidade assistencial. A Comissão, portanto, implementou e monitorou as boas práticas para assistência mais segura baseada na gestão de riscos, apontando como limitação a resistência por parte de profissionais de saúde.

Palavras-chave: Segurança do Paciente, Gestão de Riscos, Acreditação, Serviços de Saúde.

\begin{abstract}
Objective: To report on the experience of the implementation of the Quality and Patient Safety Commission as a strategy to promote quality of care. Methods: This is an experience report, with a critical-reflexive approach, about the implementation of the Patient Quality and Safety Commission in a hospital in the southwestern region of Goiás between 2012 and 2017. Experience report: The study demonstrates the feasibility of adopting the committee as a strategy to safety practices, especially in what concerns the better interaction between the teams, management of indicators, and also, the awakening of the commitment of the professionals with the execution of the service in the criterion quality assistance. The Commission has implemented and monitored good practices for safer care based on risk management, limiting resistance by health professionals.
\end{abstract}

Key words: Patient Safety, Risk Management, Accreditation, Health Services.

\section{RESUMEN}

Objetivo: Informar la experiencia de la implantación de la Comisión de Calidad y Seguridad del Paciente como estrategia para promover la calidad asistencial. Métodos: Se trata de un relato de experiencia, de enfoque crítico-reflexivo, acerca de la implantación de la Comisión de Calidad y Seguridad del Paciente en

${ }^{1}$ Faculdade de Enfermagem. Universidade Federal de Goiás, Goiânia. Brasil. *E-mail: thaquali@gmail.com 2 Universidade Estadual de Goiás, Santa Helena, Goiás, Brasil.

Financiado pelo Conselho Nacional de Desenvolvimento Científico e Tecnológico - CNPQ. 
un hospital en la región suroeste de Goiás en el período de 2012 a 2017, Relato de experiencia: El estudio demuestra la viabilidad de adoptar la comisión como estrategia a las prácticas de seguridad, principalmente en lo que se refiere a la mejor interacción entre los equipos, gestión de indicadores, y, al despertar del compromiso de los profesionales con la ejecución del servicio en el criterio calidad asistencial. La Comisión implementó y monitoreó las buenas prácticas para una asistencia más segura basada en la gestión de riesgos, apuntando como limitación a la resistencia por parte de los profesionales de la salud.

Palabras clave: Seguridad del Paciente, Gestión de Riesgos, Acreditación, Servicios de Salud.

\section{INTRODUÇÃO}

Entende-se por gestão de riscos a aplicação sistêmica e contínua de políticas e condutas na identificação, análise, avaliação, comunicação e controle de riscos e eventos adversos que afetam a segurança do paciente (ANVISA, 2013).

O Gerenciamento de riscos é um processo analítico, preventivo e normativo para melhorar o desempenho nas organizações de saúde e subsidiar a tomada de decisão por parte dos gestores, inclusive no monitoramento dos indicadores de qualidade. Entretanto, a cultura da gestão de riscos é vista como fator crítico oriundo do processo lento no tocante às melhorias estruturais e devido ao paradigma da punição, acusação do erro e subnotificações (SIQUEIRA et al., 2015).

A existência de comissões hospitalares se destaca como estratégia para o gerenciamento de riscos e por consequência melhoria contínua nos serviços de saúde. Estas subsidiam as ações da alta gestão, colaboram para a mudança da cultura de segurança, apoiam a implantação de protocolos e diretrizes provocando resultados positivos nas instituições (MARTINS et al., 2012; CAUDURO et al., 2015).

A Comissão Hospitalar representa uma ferramenta de gestão, e tem características de proatividade, com espaço aberto junto à gestão, propondo atitudes preventivas e corretivas e assim, legitimar seu papel no hospital ao realizar sistematicamente análises críticas, monitoramentos, e formulações dos fluxos e processos de trabalho com foco em resultados (MARTINS et al., 2012).

No que tange a qualidade do serviço prestado em uma unidade hospitalar, revela-se importante a magnitude dos incidentes e danos aos pacientes assistidos em hospitais. Neste interim, no Brasil em 2013, foi promulgada a Portaria o 529 que instituiu o Programa Nacional de Segurança do Paciente (PNSP), que dentre seus objetivos destaca-se: "promover e apoiar a implementação de iniciativas voltadas à segurança do paciente em diferentes áreas da atenção, organização e gestão de serviços de saúde, por meio da implantação da gestão de risco e de Núcleos de Segurança do Paciente (NSP) nos estabelecimentos de saúde" (MINISTÉRIO DA SAÚDE, 2013).

O Núcleo de Segurança do Paciente (NSP) é uma instância do serviço de saúde que deve promover e apoiar a implementação de ações voltadas à segurança do paciente, consistindo em um componente chave para a gestão de riscos, na busca pela qualidade das atividades em saúde (ANVISA, 2013).

Adicionalmente, têm se intensificado os debates sobre gestão e qualidade assistencial nos hospitais, obtendo na acreditação o desenvolvimento de abordagens dialógicos e interdisciplinares em prol ao gerenciamento do cuidado (AZEVEDO et al., 2018).

A acreditação hospitalar é um método de avaliação que busca garantir a qualidade da assistência por meio de padrões previamente definidos, assim como, importante ferramenta de gestão institucional cujo caráter é eminentemente educativo, com enfoque na melhoria contínua (ORGANIZAÇÃO NACIONAL DE ACREDITAÇÃO, 2018). 
O presente estudo vem relatar a experiência da implantação da Comissão da Qualidade e Segurança do Paciente em um hospital, que se faz importante diante da necessidade de auxiliar as equipes de saúde e Núcleos de Segurança do Paciente nas vivências práticas e estratégicas de segurança, para alcançar a qualidade assistencial. O referido trabalho foi autorizado pela Instituição e foi retirado do trabalho de conclusão de curso de MBA em gestão da qualidade em 03 de julho de 2017.

\section{RELATO DE EXPERIÊNCIA}

Trata-se de um relato de experiência, de abordagem crítico-reflexiva, acerca da implantação da Comissão da Qualidade e Segurança do Paciente em um hospital público de um município na região sudoeste do Estado de Goiás, durante o período de 2012 a 2017.

O processo de implementação da Comissão da Qualidade e Segurança do Paciente ocorreu em um hospital situado na região sudoeste do Estado de Goiás, inaugurado em 2010, por iniciativa do Governo Estadual e encontrava-se sob a gestão de Organização Social de Saúde (OSS). Foi certificada no nível 1 pela Organização Nacional de Acreditação em Outubro de 2014.

Em 2011, o hospital traçou as estratégias iniciais em prol a qualidade da prestação de serviços de saúde pautadas nos critérios da Organização Nacional de Acreditação (ONA), certificação pleiteada por esta instituição.

Para tal, contaram com apoio e capacitação de consultores externos, que por meio do processo interativo com os profissionais da instituição, auxiliaram no processo de tomada de decisão (OLIVEIRA, 2010). Ademais a decisão da alta direção foi primordial para o processo da qualidade e segurança do paciente.

Com o suporte dos consultores, e na tentativa de estar condizente com os critérios do nível 1 da acreditação, que preza pela gestão de riscos e segurança do paciente, optou-se pela constituição de uma Comissão para promover e apoiar a implementação de ações voltadas ao gerenciamento de riscos, hierarquicamente subordinada ao Núcleo de Gestão da Qualidade. Neste interim, em Outubro de 2012 implantou-se a denominada "Comissão de Riscos", que foi substituída por Comissão da Qualidade e Segurança do Paciente (CQSP) em 2013.

Figura 1: Mudança do Núcleo e Comissão de Segurança do Paciente.

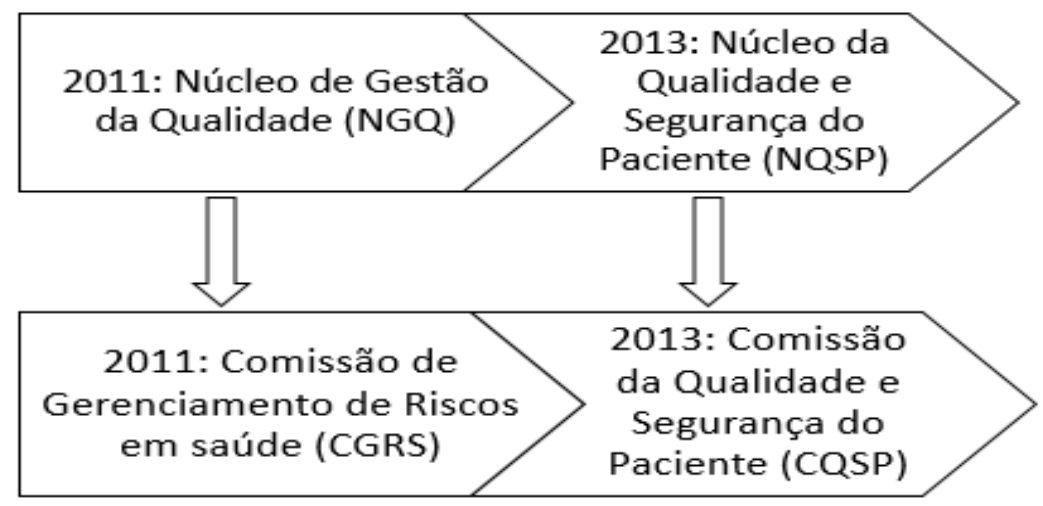

Fonte: Dados da pesquisa, 2013.

A CQSP foi constituída por uma equipe multiprofissional, composta por médico farmacêutico clínico, supervisores de enfermagem, gerente, analista e auxiliar administrativo da qualidade, biomédico, fisioterapia, psicólogo hospitalar, terapeuta ocupacional, nutrição, técnico da engenharia clínica e todos os 
diretores da instituição. Para tal, foi expresso por meio de ato administrativo a constituição da comissão, e divulgada para toda a equipe por meio de reuniões entre as lideranças, murais de avisos e sensibilizações in loco.

A CQSP foi submetida a norma institucional do funcionamento das comissões, sendo padronizada a realização de reuniões mensais que foram registradas em atas, assinadas por todos os presentes e arquivadas sob responsabilidade da equipe do Núcleo de Segurança do Paciente, seguindo orientações da ANVISA.

As reuniões ocorreram no espaço físico denominado "Sala de Reuniões" e contava com mesa redonda, cadeiras, computador com acesso à internet. Havia ainda, acesso ao flip chart, impressora, entre outros mecanismos de comunicação e estrutura para execução da reunião. As reuniões versavam sobre o desenvolvimento da qualidade da assistência no hospital, principalmente ao analisar os eventos adversos, bem como, se configurou no elo entre a direção e as estratégias de segurança do paciente favorecendo os resultados mais significativos para a gestão de riscos e acreditação hospitalar.

A CQSP foi presidida pelo diretor geral da instituição e coordenada pela gerência do Núcleo de Segurança do Paciente. O coordenador da comissão, tinha por função estabelecer ordem do dia e distribuição de tarefas para os membros, enquanto ao presidente convocar e presidir as reuniões, assinar documentações criadas pela CQSP e representar o Núcleo junto à direção do Hospital. O secretário, tinha por responsabilidade comunicar, secretariar, registrar as reuniões além de arquivar a documentação da CQSP.

Ao enfermeiro da Comissão competia gerenciar os manuais de Quedas/Lesão por pressão/Identificação do paciente/ Boas práticas pra administração de medicamentos/Cirurgia Segura; discutir e analisar as notificações envolvendo a equipe de enfermagem. Ao farmacêutico, discutir e analisar as farmacovigilâncias em consonância com a comissão farmacoterapêutica; auxiliar no gerenciamento das boas práticas para administração de medicamentos. $\mathrm{E}$ aos demais membros ou aos convidados para alguma reunião incumbiam comparecer às reuniões; colaborar com os trabalhos, quando solicitados; auxiliar no gerenciamento dos protocolos/manuais de segurança do paciente; relatar ocorrências, proferir voto ou pareceres e manifestar-se a respeito de matérias em discussão e participar da implementação dos protocolos/manuais.

Para o sucesso do planejamento das atividades da comissão, a equipe foi capacitada por consultores, em conceitos de melhoria da qualidade, segurança do paciente, ferramentas da qualidade e gestão de riscos em serviços de saúde. A capacitação dos membros da CQSP ocorreu durante a jornada de trabalho, evidenciadas em lista de presença encaminhadas ao Núcleo de Educação Permanente.

Para além das capacitações, as ações empreendidas tiveram enfoque no manejo dos protocolos de segurança do paciente, como também articulações entre as equipes multidisciplinares com vistas ao desenvolvimento participativo nos processos assistenciais.

As atividades desenvolvidas pela comissão tiveram como meta despertar o interesse das equipes e comprometê-las com a execução do serviço com base na dimensão da qualidade assistencial, almejando também ser certificado pela Organização Nacional de Acreditação (ONA). Estas ações foram estratégias chave para a melhor adesão e amadurecimento da cultura de segurança do paciente e a conquista da acreditação nível 1 em 2014.

A fim de alcançar o envolvimento institucional, os membros da comissão aplicaram junto a suas equipes de trabalho e Núcleo de Segurança do Paciente, ferramentas da qualidade para análise dos incidentes relacionados à segurança do paciente, como Diagrama de Ishikawa e Brainstorming, que possibilitaram ver zonas de riscos, suas possíveis causas para a melhoria da segurança do paciente. Em seguida os membros da CQSP junto as equipes traçaram planos de ações utilizando a ferramenta $5 \mathrm{~W} 2 \mathrm{H}$. 
A Comissão da Qualidade e Segurança do Paciente também promoveu ações para a mitigação do risco no serviço de saúde por meio de notificação de incidentes informatizado, assim como a análise proativa de riscos aplicando-se a ferramenta FMEA.

Neste ínterim, as atividades que mais resultaram em envolvimento das equipes de trabalho foram as técnicas em grupo, por meio de jogos interativos e quiz, com o objetivo de treinar as equipes sobre os conceitos da acreditação, gestão de riscos, planejamento estratégico e mapeamento dos processos. Além disso, campanhas de sensibilização com foco na prevenção de eventos adversos e notificação dos incidentes foram realizadas em todo o hospital e em todos os turnos. Ademais, a socialização das informações institucionais se deu pelos informativos mensais, intranet, homepage do hospital e nas reuniões de liderança.

A auditoria é classificada conforme a finalidade do processo (GARCIA et al., 2018). Sendo assim, a auditoria interna de risco hospitalar, cuja abordagem é para avaliar e melhorar a eficácia da gestão de riscos, controle e governança dos processos (HANSKAMP-SEBREGTS et al., 2013; ANDRADE; SICHESKI, 2017) foi uma prática implementada junto a Comissão da Qualidade e Segurança do Paciente. Esta ocorreu quadrimestralmente seguindo os critérios do manual da ONA, legislações, diretrizes institucionais, contribuindo para um melhor desempenho institucional e evolução na gestão de riscos. A auditoria interna foi executada com o apoio de auditores internos, cujos membros eram os mesmos da Comissão da Qualidade e Segurança do Paciente.

Neste sentido a comissão da qualidade e segurança do paciente contribuiu para alcançar os melhores resultados institucionais, requerendo o compromisso e envolvimento das equipes, uma vez que os processos vitais compreendem muitas pessoas, unidades, serviços e processos.

\section{EVOLUÇÃO DA COMISSÃO DA QUALIDADE E SEGURANÇA DO PACIENTE}

Como resultado de sua atuação, a CQSP definiu no primeiro ano de auditoria interna, 335 ações após a auditoria de riscos obtendo $85 \%$ de conclusão e já no último ciclo ( $9^{\circ}$ ciclo), foram planejadas 107 ações sendo $87 \%$ concluídas. Observou-se a evolução nos resultados das probabilidades dos riscos assistenciais de provável à raro, evidenciando efetividade das ações executadas, tais como: protocolos de segurança do paciente, interação de processos, notificação de incidentes, visita interdisciplinar, planejamento terapêutico e educação permanente. A auditoria interna de riscos ocorreu em 12 unidades assistências no primeiro e o segundo ciclo, e posteriormente em 17 unidades, evidenciando as melhorias na capacidade da CQSP executar auditorias internas.

A Comissão da Qualidade e Segurança do Paciente teve por finalidade alcançar efetivamente o envolvimento de todos os colaboradores e uma maior consciência para os riscos, promover a avaliação sistemática e documentada dos riscos com foco nos processos internos, definir e promover políticas, procedimentos e métodos de controle dos processos, e a promoção da Gestão do Risco fomentando as notificações voluntárias de eventos adversos.

Para tal, esta comissão trabalhou em integração com outras comissões consultivas tais como: Comissão de Controle de Infecção $(\mathrm{CClH})$ e Epidemiologia, Comissão de Resíduos, Comissão de Óbitos, Comissão de Revisão de Prontuários, Comissão de Farmácia e Terapêutica, Comissão de Ética Médica e da Enfermagem, Comissão de Demandas do Serviço de Atenção ao Usuário, Comissão de Terapia Nutricional e Comissão de Prevenção e Tratamento de Lesões de pele.

A comissão implementou a cultura de segurança, apoiou a equipe assistencial na execução das boas práticas, promoveu a notificação dos incidentes de segurança do paciente, assim como o aprendizado por meio das análises das notificações. 


\section{DISCUSSÃO}

Conforme recomendação da Agência Nacional de Vigilância Sanitária (2016), a instituição deve fornecer suporte para as ações voltadas a segurança do paciente. Neste sentido, a Comissão da Qualidade e Segurança do Paciente teve apoio estrutural para realizar as reuniões e promover as atividades planejadas.

Serra et al. (2016) apontaram como fragilidade na atuação de comissões hospitalares, as ações descontinuadas ou falta de plano de trabalho, a não priorização das atividades da comissão e ainda, a inadequada instituição da comissão. Aspecto este não condizente com a comissão em questão que teve apoio em sua implementação.

Estudos apontam que o gerenciamento do risco pode apoiar os gestores de serviços hospitalares a melhorar a segurança nas instituições, uma vez que qualidade e segurança são atributos indissociáveis (FRANÇOLIN et al., 2015). Estudo sugere que as intervenções promovidas por comissões buscam o cumprimento dos protocolos assistenciais impactando na mudança da cultura de segurança nas instituições hospitalares (CAUDURO et al., 2015). Assim, a Comissão da Qualidade e Segurança do Paciente, por meio das capacitações, sensibilização e envolvimento das equipes no gerenciamento de riscos, buscou garantir boas práticas institucionais, corroborando com estudos apontados.

Evidenciou-se que a busca da qualidade e segurança assistencial só é possível com o desenvolvimento da cultura de segurança com o comprometimento institucional, a disseminação dos conceitos de segurança do paciente entre a equipe profissional, com a adoção das práticas profissionais seguras, promoção dos processos de gestão de risco, integração de todos os processos do cuidado articulados aos processos organizacionais, responsabilização, sensibilização e capacidade de reagir a mudanças (SILVA-BATALHA; MELLEIRO, 2015).

A CQSP e o Núcleo de Segurança do Paciente, portanto, desenvolveram o papel de provocador de olhares críticos, de todos os profissionais da instituição, sobre as práticas em saúde, permitindo atuação direta dos profissionais na construção do conhecimento, contínua aprendizagem e melhoria dos processos, conforme estudo apontado por Adamy et al. (2017).

Os resultados do monitoramento e avaliação de comissões também implicaram em ações de Educação Permanente ao problematizarem as vivências do trabalho (SERRA et al., 2016), além de implementar o processo de conhecimento interdisciplinar.

A evolução dos resultados deveu-se também a gestão compartilhada, que permitiu aproximação da alta gestão com a equipe operacional, proporcionando um atendimento seguro, assim como as ações educativas com abordagem multiprofissional fortalecendo as estratégias para promoção da segurança do paciente e gestão de riscos, por meio da visualização das barreiras para segurança do paciente (OLIVEIRA et al., 2014; OLIVEIRA et al., 2017). Estudo retrata o comprometimento dos líderes e liderados como relevante para se ter assistência com mínimo de riscos (LIM et al., 2014; BERNARDES et al., 2015).

A existência de uma Comissão de Segurança do Paciente, formada por uma equipe multidisciplinar e a adoção de metodologias de análise de riscos, foi estratégia eficaz para prevenção dos eventos adversos, ao analisar e compreender os erros para evitar sua repetição (SILVA et al., 2011).

Em suma, o processo de implantação da Comissão da Qualidade e Segurança do Paciente possibilitou envolver a equipe em prol da segurança do paciente, como também alcançar a meta da acreditação hospitalar por meio do desenvolvimento de pessoas com treinamentos para a prática segura e aprimoramento na cultura de segurança e gestão de riscos. Destaca-se a interação entre as diferentes áreas do hospital e a articulação com outras comissões impactante para a melhoria da segurança do paciente. Pode-se afirmar que a experiência da implementação da Comissão mostrou-se positiva, sendo perceptível os benefícios das estratégias. Entretanto, este relato aponta a necessidade de aprimorar a 
gestão da qualidade e segurança do paciente na perspectiva da prática baseada em evidências, isto é, na possibilidade de avaliar esta experiência sob um método estruturado. Como fator dificultador salienta-se a resistência inicial por parte de alguns colaboradores, o incipiente envolvimento da equipe médica e a inexperiência das lideranças quanto à gestão de riscos e princípios da qualidade e segurança do paciente.

\section{AGRADECIMENTOS E FINANCIAMENTO}

Agradeço a diretoria da instituição hospitalar que permitiu o desenvolvimento deste estudo, assim como o Conselho Nacional de Desenvolvimento Científico e Tecnológico - CNPQ, por fomentar a publicação deste com o número de concessão.

\section{REFERÊNCIAS}

1. ADAMY EK, et al. Tecendo a educação permanente em saúde no contexto hospitalar: relato de experiência. Revista de Enfermagem do Centro-Oeste Mineiro. 2017;7:e1615.

2. ANDRADE FB, SICHESKI SJ. Auditoria interna hospitalar: Uma atividade de apoio à tomada de decisão. Revista Espacios. 2017;38(24):13.

3. ANVISA - AGÊNCIA NACIONAL DE VIGILÂNCIA SANITÁRIA. RDC n 36, de 01 de Abril de 2013. Dispõe sobre os Requisitos de Boas Práticas de Funcionamento para os Serviços de Saúde. Brasília; 2013.

4. ANVISA - AGÊNCIA NACIONAL DE VIGILÂNCIA SANITÁRIA. Implantação do Núcleo de Segurança do Paciente em Serviços de Saúde - Série Segurança do Paciente e Qualidade em Serviços de Saúde/Agência Nacional de Vigilância Sanitária. Brasília; 2016.

5. AZEVEDO CS, et al. Entre protocolos e sujeitos: qualidade do cuidado hospitalar em um serviço de hematologia. Cadernos de Saúde Pública. 2018; 34(6):e00043817.

6. BERNARDES A, et al. Implementation of a participatory management model: analysisfrom a political perspective. Journal of Nursing Management. 2015;23(7):888-897.

7. CAUDURO FLF, et al. Cultura de segurança entre profissionais de centro cirúrgico. Cogitare Enfermagem. 2015;20(1):129-138.

8. FRANÇOLIN L, et al. Gerenciamento da segurança do paciente sob a ótica dos enfermeiros. Revista Escola de Enfermagem da USP. 2015;49(2):277-283.

9. GARCIA TT, et al. Processo de auditoria em enfermagem e suas dimensões na assistência ao paciente: uma revisão sistemática de literature. Revista Eletrônica da FAINOR. 2018;11(1):1-30.

10. HANSKAMP-SEBREGTS M, et al. Efeitos das auditorias de segurança do paciente no cuidado hospitalar: desenho de uma avaliação baseada em métodos mistos. BMC Health Serv Res. 2013;13(1):2-11.

11. LIM RBT, et al. Evaluation of the Patient Safety Leadership Walkabout programme of a hospital in Singapore. Singapore Med J. 2014;55(2):78-83.

12. MARTINS C, et al. Comissões Hospitalares: A Produção De Indicadores De Gestão Hospitalar. RGSS. 2012;1(1): 97-107.

13. MINISTÉRIO DA SAÚDE. Portaria $n^{\circ} 529$, de 1ำ de abril de 2013. Institui o Programa Nacional de Segurança do Paciente (PNSP). Brasília; 2013.

14. OLIVEIRA JLC, et al. Facilitating factors in the implementation of patient safety strategies: a descriptive exploratory study. Online. braz. j. nurs. 2017;16(2):108-118.

15. OLIVEIRA RM, et al. Strategies for promoting patient safety: from the identification of the risks to the evidencebased practices. Escola Anna Nery. 2014;18(1):122-129.

16. OLIVEIRA DPR. Manual de consultoria empresarial. 6 ed. Editora Atlas, São Paulo, 2010.

17. ORGANIZAÇÃO NACIONAL DE ACREDITAÇÃO - ONA. Manual Brasileiro de Acreditação-Organizações Prestadoras de Serviços de Saúde São Paulo: ONA; 2018.

18. SERRA JN, et al. Situação dos hospitais de referência para implantação/funcionamento do Núcleo de Segurança do Paciente. Cogitare Enfermagem. 2016;21(esp.):01-09.

19. SILVA AEBC, et al. Adverse drug events in a sentinel hospital in the State of Goiás, Brazil. Revista LatinoAmericana de Enfermagem. 2011;19(2):378-386.

20. SILVA-BATALHA EMS, MELLEIRO MM. Patient safety culture in a teaching hospital: differences in perception existing in the different scenarios of this institution. Texto e Contexto - Enfermagem. 2015;24(2).

21. SIQUEIRA CL, et al. Gerenciamento de risco: percepção de enfermeiros em dois hospitais do sul de Minas Gerais, Brasil. Revista Mineira de Enfermagem. 2015;19(4):919-926. 